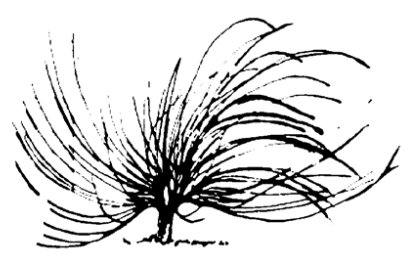

\title{
Guía para educadores perdidos en la ciudad global
}

\author{
Immanuel Cruz Fuentes ${ }^{1}$ \\ Escuela Ciencias del Movimiento Humano y Calidad de Vida \\ Universidad Nacional \\ Costa Rica \\ immanuel.cruz.fuentes@una.cr
}

\begin{abstract}
Resumen
Este ensayo es una guía metafórica para ayudar a que los educadores de la actualidad puedan circular a través de la ciudad global en la cual habitamos en el siglo XXI. Como parte del recorrido, se encaminará, seguramente, al lector por aquellas "zonas" de la gran aldea global en las cuales la educación se ha desarrollado, al permitirle que conozca sitios de interés para la teoría pedagógica de la actualidad, locales que debe visitar para entender cómo las tecnologías virtuales/digitales han cobrado importancia, a la vez que, han desplazado la experiencia física/ presencial, así como conocer puntos de encuentro para compartir nuestras experiencias didácticas. Finalmente, se realizará un paseo en el cual se hablará de los atajos que el autor ha descubierto para movilizarse en medio de la ciudad global, cómo aprender a disfrutar el escenario de la educación que se nos presenta, y qué podría interpretarse del horizonte que nos espera en el futuro de la enseñanza y el aprendizaje del siglo XXI.
\end{abstract}

Palabras clave: Docencia, educación, globalización, guía

\section{c) (i) $९$}

Recibido: 9 de mayo de 2020. Aprobado: 1 de junio de 2021

http://dx.doi.org/10.15359/rep.16-2.2

1 Académico e investigador universitario en áreas de enseñanza deportiva, biomecánica, anatomía y mediación en las ciencias; máster en Movimiento Humano y Salud Integral, UNA; doctorando en Educación, UNED; código ORCID: https://orcid.org/0000-0003-3335-8079 


\begin{abstract}
This essay is a metaphorical guide to help the educators of today to circulate through the global city in which we live in the 21 st century. As part of the tour, the reader will be directed safely to those "zones" of the great global village in which education has been developed, allowing them to know places of interest of the current pedagogical theory, spots that they must visit to understand how virtual/digital technologies have become important; at the same time, they have displaced the physical/face-to-face experience and meeting points to share our didactic experiences. Finally, we will take a walk, in which the author will explain the shortcuts that he has discovered to mobilize in the midst of the global city, how to learn to enjoy the view of education that is presented to us, and what could be expected from the horizon that we can see for the future of teaching and learning in the 21 st century.
\end{abstract}

Keywords: education, globalization, guidebook, teaching profession

\title{
Introducción
}

$\mathrm{V}$

ivimos en la era del acceso tecnológico y la información, somos una aldea global evolucionada, y tenemos nuevos medios para la comunicación, como nunca en la historia; sin embargo, también nos encontramos atrapados en la ciudad de las contradicciones, con terribles brechas entre los pudientes y los desposeídos, en una confusa realidad sociocultural en la que se nos propone que la información y el conocimiento están al alcance de todos, para así, atraer a más personas a la "urbe", pero que solo aquellos que pagan cuotas astronómicas les pueden obtener de fuentes confiables ${ }^{2}$ y seguras; lo que genera que las personas sedientas de saber, solo logren saciarse con noticias falsas, promociones y anuncios.

Entonces mis queridos docentes, al tener eso en cuenta, es normal que nos sintamos confundidos y desubicados en estos atribulados tiempos, he preparado esta guía de la "ciudad global" para que puedan orientarse, educativamente, por caminos que les resulten cómodos, seguros y por qué no, felices.

2 Pensemos en los grandes nombres de journals, revistas, bases de datos y otros. 
En este documento están señalizados los sitios de interés en las diferentes corrientes de pensamiento pedagógicas actuales, estas les podrán ayudar a ubicarse, en caso de que una vez más estén un poco extraviados en su andar. Asimismo, descubriremos que hay opciones de locales famosos en sitios estratégicos que los cambios científico-tecnológicos han construido en la educación, y si más bien, están interesados en un tour de tipo nutritivo (para su labor profesional), podrán ubicar fácilmente en el reverso, la lista de puntos de encuentro, donde podrán abordar temáticas vigentes en la didáctica educativa del siglo XXI. Por último, veremos demarcados los caminos, que este autor considera más adecuados para llegar a buen término en procesos educativos. Espero que mi guía les sirva para sentirse un poco menos perdidos.

\section{Sitios de interés: las teorías que les sugiero conocer y las corrientes por las cuales pueden dejarse llevar ${ }^{3}$}

No es ningún secreto que la práctica de la docencia es vivencial, y que por mucho que nos formemos en el ámbito formal (magisterios, institutos, universidades y otros), para muchas de las situaciones que experimentamos en nuestro quehacer profesional jamás nos pudimos (o pudieron) haber preparado (Ferreiro, 2006). Es por esto, que considero importante indicarles que en la actualidad hemos dejado de referenciarnos, de forma principal, con las señales de los modelos conductistas, que si bien es cierto han sido muy útiles otrora para establecer los caminos de la "construcción del mundo educativo", han tendido a generar una dinámica de tránsito por la vida un poco aburrida y limitante, en términos de abordajes integrales y humanizantes, para quienes nos formamos en este modelo, debido a que nos enseñaron a caminar solo por una senda preestablecida de causalidad, que no da espacio para plantearnos formas diferentes de observar nuestro mundo. Además, hay que reconocer que también han servido para permitir que ciertos grupos de personas, de notable poder, aprovechen nuestras dinámicas preestablecidas (por caminos que ellos mismos diseñaron) para establecerse en los lugares más cómodos y mejor ubicados dentro de la estructura socioeconómica (Casassus, 2002; McLaren, 2015).

Actualmente, esto lo hemos intentado cambiar, pues hemos venido reflexionando mucho sobre nuestros caminos, y entendemos que

3 Educativamente hablando, claro está. 
la ruta de educar no se asienta, de forma exclusiva, en dictar cátedra desde una falsa posición elevada, sino más bien, está en poder compartir el trayecto con nuestros estudiantes, para así no solo ayudarles en su propio proceso de autodefinición como transeúntes del mundo, sino también aprender ${ }^{4}$ sobre nosotros mismos y nuestras elecciones de vida, a la vez que potenciamos y reconstruimos la educación en sí (León, 2016). Desde esta perspectiva tan transformadora, entendemos que la educación como ciencia se ha reconfigurado, y ahora acepta, más allá de un positivismo impuesto, que hay otras formas de crear conocimientos científicos ${ }^{5}$, hecho por el cual mencionaré, específicamente, locaciones cuyas ideas fueron gestadas desde el constructivismo que, como corriente filosófica educativa, propone una revisión de la enseñanza para permitir el aprendizaje de tipo significativo y benéfico en los individuos, desde lo que nace en ellos mismos, un saber verdaderamente personal (Mercer, 1997; Reyero, 2019).

Veremos entonces, que hay tres propuestas que a este autor le parecen particularmente rescatables para que ustedes puedan visitar, y tal vez así, se ayuden a entender mejor esta metafórica ciudad educativa de la que les hablo:

Pedagogías sociocríticas: esta locación requiere una mente más abierta, sensible y empática con el prójimo, pues no parte de la idea de "la casa que les gustaría para ustedes mismos", sino de "la casa en la que le gustaría que viviese esa otra persona con la que se encuentran cuando caminan por la calle". Nacida desde las observaciones sociales de arquitectos (educativos) como el afamado Paulo Freire (Britto, 2008), basa su proyecto en el despertar de la conciencia crítica sobre el entorno (comunidad) de cada individuo en diferentes niveles, para así permitir que aquellas personas sin una casa, tengan la posibilidad de construirla por sí mismas, en un acto de autodeterminación y libertad (Freire, 1970, 1985). Esto propone también la validación de saberes diversos, por lo cual el trillo del barrio ahora puede ser tan importante como la autopista de la transnacional, y el diálogo abierto y humanizante guía las dinámicas entre sus residentes; sin embargo, no se deja de lado la discusión con el sistema y la crítica constructiva y abierta sobre

4 Sí, como personas que enseñamos, es importante aprender de cuando en cuando, yo mismo lo intento ocasionalmente.

5 En nuestro caso, de tipo pedagógico. 
las necesidades diversas, en las vidas de las personas (Lloyd y Bahr, 2010; Fassett y Warren, 2007; Lorente, 2013).

Pedagogías holísticas: ubicadas en un barrio diverso y colorido, esta construcción reciente se basa en la aceptación de la integralidad humana, $\mathrm{y}$, por ende, de su incorporación en los procesos educativos de aspectos espirituales, cognitivos, físicos, psicológicos y emocionales en la formación de las personas (Chávez et al., 2009; Educación de Alta Conciencia, 2013). Aquí, encontraremos interesantes actividades y dinámicas que abogan por comprender que toda persona que podamos conocer, es más que solo un cúmulo de conocimientos útiles, pues su valor radica en la gran cantidad de elementos (psíquicos, emocionales, físicos, sociales, y otros) que lo componen como un ser pensante y sensible. La educación para la paz o desde la ternura se puede sentir en este lugar, y permite abordar las situaciones de la enseñanza y el aprendizaje desde una visión muy completa que no rechaza el ambiente, sino que lo incorpora desde múltiples dimensiones para buscar un desarrollo pleno de la persona que, por aquí decide y puede caminar (Doin et al., 2012; Educación de Alta Conciencia, 2013).

Pedagogía de la complejidad: el más laberíntico de los sitios de interés de la actualidad (no por nada se le llama así), y al mismo tiempo de los más esclarecedores; según le han informado a este autor, es de los lugares más recientemente abordados por nosotros los educadores. Su propuesta arquitectónica está basada en la integración de la intersubjetividad para la creación de los conocimientos en los procesos de aprendizaje (Calzadilla, 2004; Sánchez y Pérez, 2011), y valora un abordaje más allá de lo integrador, llegando a componer obras y creaciones de todo tipo desde todas las perspectivas posibles. La realidad es que aún hay mucho por aportar en la construcción de este modelo, pero les recomiendo que valoren dar un paseo por este lugar para generar sus aportes y ver cómo pueden establecer en su haber docente esta visión. Me permito, de manera particular, recomendarles el ayudar en la elaboración de tejidos de conocimientos para que así puedan entender y valorar la multiplicidad de saberes transdisciplinarios que pueden involucrarse en la educación humana (Morin, 2002; Sánchez y Pérez, 2011).

Ahora mis queridos educadores, se preguntarán ustedes: ¿cómo puedo calzar esto con las dinámicas actuales de mis procesos de enseñanza? ¿en qué momento empiezo a hacer "click" y tocar la pantalla táctil? A eso vamos. 


\section{Locales famosos: tecnologías, avances y retrocesos científicos en nuestro andar educativo ${ }^{6}$}

Estos locales representan puntos de inflexión en la "aldea global", son cambios tecnológicos que nos obligaron a repensar nuestras formas y contenidos en muchos planos, pues la tecnología es el uso aplicado de los conocimientos creados científicamente, los cuales se caracterizan por lo metódico y sistemático de sus procesos en todas sus fases (Bunge, 2001).

En términos de la educación, la tecnología ha generados gran cantidad de aportes, sin los cuales, en la actualidad no sería posible enfrentar los retos de ser docentes, desde las tizas y los marcadores acrílicos con sus respectivas pizarras, hasta la digitalización de documentos o la elaboración de guías educativas audiovisuales para la consulta asincrónica (Martí, 2017).

Particularmente, me permitiré referenciar dos lugares importantísimos, los cuales están en boga y se han vuelto necesarios en la última década, los repositorios virtuales de información y la videoconferencia, en estos sitios, podrán conseguir muchos insumos necesarios para desarrollarse como docentes en el nuevo milenio.

Repositorios virtuales de información ${ }^{7}$ : como la famosa analogía del sistema operativo de Microsoft (www.microsoft.com) que se llama "Windows", ahora las impresiones, los blocs de notas, los calendarios y muchas otras herramientas laborales de las oficinas se han digitalizado o virtualizado. No es la excepción para los archivos de materiales educativos que se usan en la actualidad, las bibliotecas se han digitalizado, los cursos con todo y sus aulas incluidas, se han estructurado en ambientes virtuales, y ahora hasta se dice que ser docente requiere de vigencia no solo en uso de los aparatos tecnológicos físicos, sino también en el desarrollo de destrezas en ambientes virtuales, en los cuales, las dinámicas y "objetos" cumplen funciones de sus homólogos del mundo real, a tal punto que la nueva situación de la educación es, sin lugar a dudas, sumamente virtual (Pallarès y Chiva, 2017; Tello y Aguaded, 2009).

\footnotetext{
6 Recuerde que siempre es usted quien decide cómo y para dónde se dirige.

7 Estas carpetas y hojas no necesitan ordenarse en gavetas ni consumir espacio físico.

8 "Ventanas" en español.
} 
La videoconferencia ${ }^{9}$ : este es el más nuevo de los locales comerciales de la globalización, la interacción humana no física, que no viene desde la realidad virtual (creaciones digitales), sino desde el pensamiento que idea que, la virtualización de la realidad puede "entrar y salir" de forma libre entre el plano físico y el de los datos. En la actualidad, el entender que se puede ver, hablar y escuchar a otros seres humanos, al mismo tiempo que es posible compartir información diversa (procesos, ideas, ejemplos y muchos otros), es solo el primer paso para optimizar los medios, a través de los cuales, damos forma a nuestros procesos de enseñanza; casi podría decirse que es un criterio básico para la docencia moderna, y que pronto será indispensable en nuestro haber para poder desenvolvernos decentemente como docente en el siglo XXI (Rodrigo-Cano et al., 2019; Cristiano, 2019).

Una vez hayan visitado estos locales, podrán analizar que su presencia ha causado un gran impacto, primero, al proponer todo un nuevo conjunto de habilidades necesarias para el desenvolvimiento efectivo del ser humano moderno, muchas ligadas a conceptos virtuales abstractos y difíciles de comprender para aquellas generaciones que no se han familiarizado con estas dinámicas; en segundo lugar, porque evidencian un desarrollo terriblemente acelerado de las herramientas, que cambian su validez y utilidad con un paso tan asolador, que hasta nos hacen cuestionarnos nuestras posibilidades de utilizarlos cómoda y de manera tan efectiva (Cristiano, 2019; Tello y Aguaded, 2009).

No tengan miedo de entrar a estos locales, pero recuerden que no deben comprar todo lo que les ofrezcan, por novedoso que sea, en ocasiones es fácil olvidar que los que educamos somos nosotros, no los software o los aparatos tecnológicos (Rebelo, 2019; Ward et al., 2017).

\section{Puntos de encuentro: las didácticas propias de nuestro tiempo, ¿qué hacemos y cómo cambiarlo ${ }^{10}$}

Si para este momento se sienten muy abrumados, no se preocupen, es normal, este es un mal que nos aqueja a la gran mayoría ${ }^{11}$ de los educadores conscientes de la actualidad, incluido este autor. Para poder aliviar un poco nuestra carga, esta guía también incluye puntos de

9 Vernos cara a cara, sin estar frente a frente.

10 Si es que usted lo considera necesario, recuerde que el cambio es parte de la vida, no se niegue esa valiosa oportunidad.

11 Si no es que a todos. 
reunión interesantes, en los cuales podremos escuchar sobre las didácticas modernas que se practican en el panorama educativo.

El diálogo es la forma de aprovechar estas oportunidades, en especial aquel que podemos entablar de forma crítica con nuestros métodos, pues tal y como señalan autores más informados que este (Chen y Kidd, 2011; Gauthier y Collard-Fortin, 2016; Nicoll y Spendlove, 2016; Visvizi et al., 2019), el verter nuestras experiencias presenciales en la virtualidad no solo es insuficiente, sino también contraproducente. La principal lección que nos está dejando el paisaje que podemos apreciar en nuestra "ciudad global" es, que las experiencias que reconocen el valor y las capacidades de nuestros congéneres son las más significativas para lograr enseñar y aprender de forma efectiva y benéfica (Reyero, 2019).

Presten atención al caminar por esta zona, pues podrán captar rumores sobre prácticas disruptivas que pretenden romper con las estructuras de aprendizaje tradicionales desde el uso de las posibilidades digitales (Chen y Kidd, 2011; García y Álvarez, 2019), las dinámicas educativas que involucran la creación de comunidades de aprendizajes autónomas que autorregulan sus procesos desde la distancia y la virtualidad (Strobel et al., 2016; Pallarès y Chiva, 2017), prácticas dialógicas desde las realidades vividas en los entornos propios de las personas involucradas (Gauthier y Collard-Fortin, 2016), e incluso adaptaciones muy válidas de los modelos constructivistas a las posibilidades tecnológicas de nuestra época (Reyero, 2019).

\section{Conclusiones de los recorridos: atajos, paseos escénicos y nuevos horizontes $^{12}$}

Creo mis queridos lectores, que ahora se encuentran ustedes mucho más claros respecto a en dónde se encuentran parados (educativamente), y hacia dónde podrían encaminarse; sin embargo, aún quisiera señalarles aquellos atajos que me han resultado cómodos como educador para transitar en esta loca ciudad que es el mundo actual:

No dejar de probar nuevas opciones: a pesar que pueda resultar espeluznante el enfrentarse a herramientas (software, tecnologías y otros) y, situaciones (ambientes, dinámicas, u otros) que no conocemos, es valioso aprender nuevas habilidades que puedan potenciar nuestra labor docente y ayudar en la construcción de conocimientos en

12 Hay tanto por descubrir aún, ¿no les emociona? 
los estudiantes (Ferreiro, 2006). Quién sabe, puede que hasta lleguen a disfrutar de estas nuevas experiencias.

Escuchar a las diferentes personas con las que se comparten los procesos de enseñanza y aprendizaje: como vimos, la mayoría de las propuestas teóricas de la educación actual hablan del intercambio de saberes y conocimientos, esto incluye las opiniones y experiencias previas de otros, verán que en tanto se apoyen en los intereses y capacidades de otros, los suyos propios se verán reflejados.

No perder la autenticidad de mis procesos: el rescate de la identidad es uno de los mayores retos en esta aldea global que busca homogenizarnos, ser nosotros mismos y sentirnos cómodos en el uso de nuestras posibilidades es sumamente importante, pues a final de cuentas, si hemos decidido vivir enseñando, hemos de hacerlo disfrutando de ser quienes somos.

Criticar de forma continua pero comprensiva mi propia prácti$c a$ : no está de más cuestionarnos, y de forma constante buscar mejorar, siempre se pueden hallar nuevos caminos que antes no conocíamos, los cuales pueden llevarnos a lugares insospechados e increíbles; eso sí, hemos de ser compasivos con nosotros mismos, y no juzgarnos excesivamente duro, no olvidemos que somos humanos.

Con esto, mis queridos lectores, concluye esta guía, espero, con sinceridad, que les sea de ayuda para poder transitar en medio de esta locura que es el tratar de enseñar y aprender en el nuevo milenio, pues como autor, debo confesarles que yo también me siento perdido.

\section{Referencias}

Britto, Z. (2008). Educación popular, cultura e identidad desde la perspectiva de Paulo Freire. En M. Gadotti, M. V. Gomez, J. Mafra y A. Fernandes de Alencar (Comps.), Paulo Freire: Contribuciones para la pedagogía (pp. 29-45). CLACSO. http://biblioteca.clacso. edu.ar/gsdl/collect/clacso/index/assoc/D1599.dir/gomez.pdf

Bunge, M. (2001). La ciencia, su método y su filosofia (4. ${ }^{\mathrm{a}}$ Ed.). Editorial Sudamericana.

Calzadilla, R. (2004). La pedagogía como ciencia humanista: conocimiento de síntesis, complejidad y pluridisciplinariedad. Revista de Pedagogía, 25(72), 123-148. http://ve.scielo.org/scielo. php?pid=S0798-97922004000100005\&script=sci_arttext 
Casassus, J. (2002). Cambios paradigmáticos en educación. Revista Brasileira de Educação, 20, 48-59. https://www.scielo.br/pdf/ rbedu/n20/n20a04.pdf

Chávez, J., Deler, G. y Súarez, A. (2009). Principales corrientes y tendencias a inicios del siglo XXI de la pedagogía y la didáctica. Educación Cubana. http://www.cubaeduca.cu/media/www.cubaeduca.cu/medias/pdf/4750.pdf

Chen, I. y Kidd, T. T. (2011). Ubiquitous learning: Strategies for pedagogy, course design, and technology. Information Age Publishing. https://books.google.co.cr/books?isbn=1617354376

Cristiano, A. (2019). Teoria crítica da tecnologia e educação: desafios contemporâneos (Tesis de maestría no publicada). Universidade La Salle - UNILASALLE. http://repositorio.unilasalle.edu.br/ bitstream/11690/1249/1/achabowski.pdf

Doin, G. (productor), Guzzo, V. (productora) y Vautista, J. (director). (2012). La educación prohibida [película documental]. Red de Educación Alternativa. https://www.youtube.com/ watch? $\mathrm{v}=-1$ Y $9 \mathrm{OqSJKCc}$

Educación de Alta Conciencia. (2013). Los errores del sistema educativo actual. Altaeducacion. https://www.youtube.com/ watch?v=cii_szAAutM

Fassett, D. L. y Warren, J. T. (2007). Critical Communication Pedagogy. SAGE Publications. https://books.google.co.cr/ books?isbn=1452279047

Ferreiro, R. F. (2006). El reto de la educación del siglo XXI: La generación N. Apertura, 6(5), 72-85. https://www.redalyc.org/ pdf/688/68800506.pdf

Freire, P. (1970). Pedagogía del oprimido. Siglo XXI.

Freire, P. (1985). La educación como práctica de la libertad. Siglo XXI.

García, A. y Álvarez, I. (2019). Educación hacker: Una expresión emergente de la pedagogía crítica para la sociedad en red. Revista Teias, 20(Edición especial), 167-183. https://doi.org/10.12957/ teias. 2019.43375

Gauthier, D. y Collard-Fortin, U. (2016). Community of Practice and its Applied Results in the Teaching of Science-Technology of the Secondary Sector. En Z. Smyrnaiou, M. Riopel y M. Sotiriou (Eds.), Recent advances in science and technology education, ranging from modern pedagogies to neuroeducation 
and assessment (pp.15-29). Cambridge Scholars Publishing. https://www.cambridgescholars.com/recent-advances-in-science-and-technology-education-ranging-from-modern-pedagogies-to-neuroeducation-and-assessment

León, A. C. (2016). Una aproximación a las discusiones en el campo de la educación y la pedagogía: Estado de la cuestión. Pedagogía y Saberes, 44, 93-103. http://www.scielo.org.co/pdf/pys/n44/ n44a09.pdf

Lloyd, M. y Bahr, N. (2010). Thinking Critically about Critical Thinking in Higher Education. International Journal for the Scholarship of Teaching and Learning, 4(2). 1-17. https://doi.org/10.20429/ ijsotl.2010.040209

Lorente, G. (2013). Reflexiones críticas y autocríticas sobre pedagogía crítica. Revista Interuniversitaria de Formación Del Profesorado, 77(27), 77-92. https://dialnet.unirioja.es/descarga/articulo/4840079.pdf

Martí, J. (2017). Educación y tecnologías (Primera Ed.). Servicio de Publicaciones de la Universidad de Cádiz.

McLaren, P. (2015). Pedagogía crítica y lucha de clases en la era del terror neoliberal. Revista Internacional de Educación Para La Justicia Social, 4(2), 29-66. https://revistas.uam.es/riejs/article/ view/2369

Mercer, N. (1997). La construcción guiada del conocimiento: El habla de profesores y alumnos (1. ${ }^{\mathrm{a}}$ Ed.). Paidós. https://books.google. co.cr/books?isbn $=8449303222$

Morin, E. (2002). La cabeza bien puesta. Ediciones Nueva Visión. https://vra.unah.edu.hn/dmsdocument/4593-la-cabeza-bien-puesta

Nicoll, B. y Spendlove, D. (2016). "Academic tasks" in design and technology education: Past, present and future. En M. J. de Vries, S. Fletcher, S. Kruse, P. Labudde, M. Lang, I. Mammes, I. ... M. Winterbottom (Eds.), Technology education today: International perspectives (pp. 125-146). Waxmann. https://books.google. co.cr/books?isbn $=3830983840$

Pallarès, M. y Chiva, O. (2017). La pedagogía de la presencia: Tecnologías digitales y aprendizaje-servicio (1. ${ }^{\mathrm{a}} \mathrm{Ed}$.). Editorial UOC.

Rebelo, M. (2019). Educação e tecnologia: a crise da inteligência [Educación y tecnología: la crisis de la inteligencia]. Educação 
(UFSM), 44, 1-22. https://periodicos.ufsm.br/reveducacao/ article/view/37943/pdf

Reyero, M. (2019). La educación constructivista en la era digital. Tecnología Ciencia y Educación, 12, 111-127. https://tecnologia-ciencia-educacion.com/index.php/TCE/article/view/244

Rodrigo-Cano, D., Aguaded, J. I. y García, F. J. (2019). Metodologías colaborativas en la Web 2.0. El reto educativo de la Universidad. REDU: Revista de Docencia Universitaria, 17(1), 229-244. https://polipapers.upv.es/index.php/REDU/article/view/10829/0

Sánchez, J. y Pérez, C. (2011). Hacia un currículo transdisciplinario: Una mirada desde el pensamiento complejo. Revista de Teoría y Didáctica de Las Ciencias Sociales, 17, 143-164. https://www. redalyc.org/pdf/652/65221619010.pdf

Strobel, J., Lancaster, M. D. y Luo, Y. (2016). US K-12 engineering education history; A keyword, field and social network analysis of trends. En M. J. de Vries, S. Fletcher, S. Kruse, P. Labudde, M. Lang, I. Mammes, I. ... M. Winterbottom (Eds.), Technology education today: International perspectives (pp. 39-58). Waxmann. https://books.google.co.cr/books?isbn=3830983840

Tello, J. y Aguaded, J. I. (2009). Desarrollo profesional docente ante los nuevos retos de las tecnologías de la información y la comunicación en los centros educativos. Píxel-Bit. Revista de Medios y Educación, 34, 31-47. https://www.redalyc.org/ pdf/368/36812036003.pdf

Visvizi, A., Lytras, M. D. y Daniela, L. (2019). The future of innovation and technology in education: Policies and practices for teaching and learning excellence. Emerald Publishing.

Ward, A. F., Duke, K., Gneezy, A., y Bos, M. W. (2017). Brain drain: The mere presence of one's own smartphone reduces available cognitive capacity. Journal of the Association for Consumer Research, 2(2), 140-154. https://doi.org/10.1086/691462 